Vol. 2, nº 1 | 1998

Varia

\title{
Mariage aristocratique et doctrine ecclésiastique : Le témoignage du rapt au Parlement de Paris pendant la guerre de Cent ans
}

\section{Geneviève Ribordy}

\author{
(2) OpenEdition \\ Journals \\ Édition électronique \\ URL : https://journals.openedition.org/chs/990 \\ DOI : $10.4000 /$ chs.990 \\ ISSN : 1663-4837 \\ Éditeur \\ Librairie Droz \\ Édition imprimée \\ Date de publication : 1 janvier 1998 \\ Pagination : 29-48 \\ ISBN : 2-600-00279-0 \\ ISSN : 1422-0857
}

Référence électronique

Geneviève Ribordy, « Mariage aristocratique et doctrine ecclésiastique : Le témoignage du rapt au Parlement de Paris pendant la guerre de Cent ans », Crime, Histoire \& Sociétés / Crime, History \& Societies [En ligne], Vol. 2, n¹ | 1998, mis en ligne le 03 avril 2009, consulté le 23 mars 2022. URL: http://journals.openedition.org/chs/990 ; DOI : https://doi.org/10.4000/chs.990 


\title{
Mariage aristocratique et doctrine ecclésiastique:
}

\author{
Le témoignage du rapt au Parlement de Paris \\ pendant la guerre de Cent ans ${ }^{1}$
}

\author{
Geneviève Ribordy²
}

T'étude du rapt comme stratégie matrimoniale permet de vérifier dans quelle

Lmesure la doctrine matrimoniale de l'Église a été assimilée par la noblesse française. Les plaidoiries des vingt-quatre procès pour rapt débattus au Parlement de Paris entre 1375 et 1453 traitent davantage du mariage conclu que de l'enlèvement. Ce faisant, elles démontrent que si la noblesse connaît parfaitement les règles du droit canon et s'en sert à son avantage, elle applique surtout les rites ecclésiastiques et reste réfractaire à l'essentiel de la doctrine. Le consentement des époux demeure subordonné à celui des parents qui orchestrent les alliances pour des raisons politiques et économiques.

The study of ravishment as a marriage strategy serves as an indicator of the assimilation of the ecclesiastical doctrine of marriage by the French nobility. In the twenty-four ravishment cases appearing before the Parliament of Paris between 1375 and 1453, marriage, not crime, is the main topic of discussion. These cases show that while nobles have a perfect understanding of the ecclesiastical rules and use them as needed, their marriage customs have only included the church rites, casting aside the essentials of the doctrine. Aristocratic weddings continue to be concluded for political and economic reasons, with parental consent overruling the spouses'.

Clé de voûte de la doctrine ecclésiastique du mariage élaborée au XII ${ }^{\mathrm{e}}$ siècle, le consentement des époux constitue une notion difficile à imposer à la noblesse médiévale ${ }^{3}$. Les enjeux économiques, sociaux et politiques des stratégies matrimoniales de 1'aristocratie se concilient mal avec l'idée que les enfants soient laissés

1 L'auteur tient à remercier Madame Claude Gauvard et Madame Denise Angers pour leur aide et leurs conseils au moment de la relecture et de la publication de cet article.

2 Geneviève Ribordy est doctorante en histoire à l'Université de Montréal. Elle termine la rédaction d'une thèse portant sur le mariage dans la noblesse française (1375-1475). Elle a notamment publié: Les prénoms de nos ancêtres. Étude d'histoire sociale, Québec, Septentrion, 1995 (prix Michel Brunet 1996, décerné par l'Institut d'histoire de l'Amérique française); «La famille en NouvelleFrance: bilan historiographique», Cahiers d'histoire, 1992, 12, 2, p. 24-50; "Le choix des prénoms à Sudbury au tournant du XX' $X^{c}$ siecle ", Revue d'histoire de l'Amérique française, 1989, 43, 2, p. 179 201.

3 La doctrine d'un mariage monogame, indissoluble, exogame et fondé sur le consentement des époux est désormais bien connue grâce à un très grand nombre d'études. Parmi les plus importantes, citons Gaudemet (1980) et (1987), Esmein (1891), Brundage (1987) et Le Bras (1927). 
libres de choisir ou de refuser leur époux. Georges Duby, le premier, a étudié cet écart entre le modèle ecclésiastique et le modèle aristocratique de mariage. Ses ouvrages, Medieval Marriage. Two Models from Twelfth Century France et Le chevalier, la femme et le prêtre, démontrent comment la distance entre les deux modèles s'est rétrécie entre le $\mathrm{X}^{\mathrm{e}}$ et le XIIr' siècle:

Au lendemain de l'an mil, (...) (l'historien) découvre, affrontées, deux conceptions du bon mariage, celle qui depuis très longtemps guidait la conduite des guerriers, celle que depuis très longtemps tentaient de faire accepter les prêtres; il perçoit que, dans un premier temps, l'une et l'autre se durcirent; vers l'an 1100, le conflit paraît atteindre sa pleine intensité; puis il s'apaise; au début du XIII siècle, (...) l'accord est fait ${ }^{4}$.

L'intégration de la doctrine ecclésiastique aux pratiques matrimoniales de la noblesse est-elle vraiment achevée au XIIr ${ }^{\mathrm{c}}$ siècle ? Aux XIV et XV siècles, l'accord est-il réellement fait? L'étude d'une stratégie matrimoniale, le rapt, nous permettra d'évaluer, pour les derniers siècles du Moyen Age, l'écart qui subsiste encore entre les deux modèles 5 .

Si dans le contexte actuel, le mot rapt désigne strictement un enlèvement, il revêt d'autres significations à l'époque médiévale. Il existe une certaine confusion dans les sources de l'époque, un aspect sexuel ou matrimonial venant souvent se greffer à la notion d'enlèvement. Ainsi, les termes rapt, ravissement, ravir, raptus et rapere mettent l'accent sur l'enlèvement; mais le mot rapt semble être utilisé au Parlement de Paris surtout pour qualifier un enlèvement suivi d'un mariage, alors que le terme ravissement désigne plus généralement un enlèvement suivi d'un viol ${ }^{6}$. Aux fins de cette étude, nous avons défini le rapt comme étant l'enlèvement d'une femme dans le but de la forcer à contracter un mariage. Cette définition est d'ailleurs conforme au droit canon, puisque le crime de rapt y dépend de trois critères: «a) il doit avoir une femme pour objet; - b) être effectué par voie de contrainte ou de dol, donc sans le consentement de celle qui en a été l'objet; si elle est mineure, sans le consentement de ses parents ou tuteurs; $-c$ ) avoir pour fin le mariage ou la satisfaction de l'appétit sexuel »?.

Le rapt débute par un acte violent, contraire aux voux de la femme qui en est victime, et se termine par un mariage. Poursuivi en justice, il donne lieu à un procès où l'on conteste davantage le mariage établi que le crime lui-même. Par conséquent, les procès pour rapt fournissent l'occasion d'étudier les critères du mariage, et surtout la place centrale qu'occupe la question du consentement dans l'ensemble des considérations qui en assurent la validité.

4 Duby (1981, p. 301-302). Voir aussi Duby (1978).

5 Très peu d'historiens ont cependant abordé le rapt à la fin du Moyen Age. En France, seule Benveniste (1990) rapporte brièvement cinq cas présentés au Parlement de Paris. Elle conclut que la pratique du rapt était ancrée dans les mours de la noblesse qui y trouvait une solution aux difficultés financières créées par la guerre de Cent Ans, par la baisse des revenus de la terre et par l'hécatombe d'Azincourt. En marge de la France, Greilsammer (1988) et (1990) consacre au rapt un chapitre de livre et un article. Elle y démontre le nombre élevé de causes de rapt parmi les jugements des tribunaux urbains. Ce sont surtout des rapts de séduction, commis par des couples amoureux désirant se marier selon leur cœur et contre la volonté de leurs parents.

6 Porteau-Bitker (1988, p. 496).

$7 \quad \mathrm{Naz}$ (1965, t. VII, col. 454). 
Les sources judiciaires permettent de bien étudier le mariage car on y retrouve un débat autour de sa légalité et de sa validité. Les cours ecclésiastiques qui détiennent théoriquement l'exclusivité des litiges matrimoniaux, ont fait connaître les déviances les plus communes aux règles matrimoniales et les points susceptibles de susciter disputes et délits. ${ }^{8}$. Les cours relevant des pouvoirs laïques livrent également les secrets du mariage car les seigneurs, en particulier les rois, ne se sont jamais résignés à abandonner à l'Église toute juridiction sur la famille et le mariage. Par conséquent, ils se sont saisis des litiges touchant à des questions financières ou à des problèmes de violence et de paix publique ${ }^{9}$. Le rapt, crime à la fois violent et lourd d'intérêts financiers, est au nombre de ces exceptions jugées par les cours laïques, entre autres par le Parlement de Paris.

En parcourant les plaidoiries des registres criminels du Parlement de Paris entre 1375 - date de leur apparition - et 1453 - fin de la guerre de Cent Ans --, nous avons trouvé la trace de vingt-quatre rapts que nous avons complétés avec les lettres et les arrêts des registres criminels du Parlement et avec les lettres de rémission conservées au Registre du Trésor des Chartes ${ }^{10}$.

Les causes relevées ne se répartissent également ni dans le temps, ni dans l'espace. Elles se concentrent particulièrement sur deux périodes: l'une située entre 1398 et 1416, 1'autre entre 1443 et $1450^{11}$. La moitié Nord de la France est la plus représentée: la Picardie avec six causes, suivie de la Touraine - cinq causes - et du Poitou - quatre causes; de l'île-de-France, de l'Anjou, de la Champagne, de la Bourgogne, de la Flandre et du Bourbonnais ne proviennent qu'une ou deux causes. Seules deux causes sont issues du Sud de la France, des provinces du Beaucaire et du Limousin. Même si les causes sont surtout originaires du Nord de la France, il ne faut pas conclure pour autant qu'il constitue le terrain de prédilection du rapt. Il faut

8 Les cours ecclésiastiques ont fait l'objet de nombreuses recherches, particulièrement en Angleterre. Voir par exemple Helmholz (1974), Ingram (1981) et (1987), Sheehan (1971). Pour la France, mentionnons Dufresne (1976), Finch (1992), Lévy (1965), Gottlieb (1980) et Lefebvre-Teillard (1973).

9 Certaines recherches se sont déjà servies des documents provenant des cours laïques pour étudier le mariage. De nouveau, la plus grande partie des recherches émanent de l'Angletere. Par exemple, Bennett (1981) et Erickson (1990). En France, ce type de source n'a pas encore suscité beaucoup de recherches. Gauvard (1991, p. 573-612) consacre un chapitre au couple dans son ouvrage sur la criminalité française de la fin du moyen âge; citons aussi le travail de Greilsammer (1990).

10 Cependant, seulement vingt rapts ont donné lieu à des fiançailles et à un mariage décrit dans les plaidoiries. Les quatre rapts sans mariage peuvent tout de même être considérés comme de véritables rapts. Deux d'entre eux sont des tentatives de rapt qui ont échoué et qui n'ont donc pas pu se solder par un mariage: il s'agit des rapts des deux sœurs Hemery, Jehanne et Jehannette (Hemery vs D. Asincourt, Arch. nat., X 2a 14, fol. $241 v^{\circ}-292 v^{\circ}$ ). Katherine la Prevoste et de Jehan Gobert Descanale se retrouvent en procès avant que leur mariage n'ait pu se conclure: le rapt a réussi, les fiançailles ont été célébrées, le couple désire s'épouser mais ne peut le faire sans une lettre de rémission car le ravisseur est poursuivi par la famille de la fille (Warisonne vs Bezon, Arch. nat., JJ 121, lettre 216 , fol. $115 \mathrm{v}^{\circ}$ ). Enfin, l'enlèvement visait clairement un mariage dans un dernier cas sans que nous puissions savoir si ce mariage a eu lieu et en quels termes (Pronnier vs Rochefort, Arch. nat., X 2a 25 , fol. $139 \mathrm{r}^{\circ}-142 \mathrm{v}^{\circ}$ ).

1 Certains registres comportent un plus grand nombre de procès pour rapt que d'autres. Le X 2 a 14 (1400-1408) - six causes -, le X 2a 17 (1410-1417) - cinq causes - et le X 2a 24 (1443-1448) - six causes - sont particulièrement riches en rapts alors que les X $2 \mathrm{a} 18$ (1422-1432) et X 2a 25 (14481453) ne contiennent qu'une cause chacun; le $X 2 a 22$ (1436-1443) n'en contient aucune. La diminution du nombre de rapts au Parlement se fait donc sentir tout de suite après Azincourt. Cette constatation contre dit l'hypothèse de Benveniste (1990, p. 21) qui suppose que le rapt constitue une solution de rechange adoptée par la noblesse pour contrevenir à la perte de revenus provoquée par les crises des $\mathrm{XIV}^{e}$ et $\mathrm{XV}^{e}$ siècles et qu'elle lie particulièrement à la bataille d'Azincourt. 
plutôt y voir l'influence de la proximité du Parlement de Paris: plus la distance est grande, plus les chances que la cause se présente au Parlement diminuent ${ }^{12}$.

Vingt-quatre causes en soixante-dix-huit ans: ce nombre peut suggérer que le rapt est rare aux XIV et $X V^{\mathrm{e}}$ siècles ${ }^{13}$. Cependant, le Parlement est la plus haute instance du pays, agissant surtout comme cour d'appel. Nombre de cas sont sans doute restés aux instances civiles inférieures - juridictions seigneuriales, cours royales des prévôts, des baillis et des sénéchaux, tribunaux urbains - et d'autres ont trouvé leur résolution devant les officialités. De nombreuses causes n'ont probablement jamais donné lieu à un procès, que la victime soit restée impuissante et incapable d'agir ou que, plus probablement, un accord entre les familles soit venu mettre un terme au conflit, évitant ainsi les lourds frais que pouvait entrâner un procès en justice.

De plus, on peut établir un lien entre le petit nombre de rapts poursuivis au Parlement de Paris et le fait qu'il n'y a pas de criminalisation du rapt. Même si les procureurs du roi s'acharnent à affirmer «que telz cas pulullent mout fors et pour ce on les doit mettre peine a les fort réprimen ${ }^{14}$, aucune ordonnance royale ne légifere contre le rapt auX XIV et $X^{\mathrm{e}} \mathrm{V}^{\mathrm{e}}$ siècles. Le roi et sa justice n'ont pas cherché à réprimer le rapt, sauf en cas de plainte de la victime et de sa famille ou sauf en temps d'affirmation du pouvoir royal. Il n'est donc pas étonnant que les ravisseurs obtiennent facilement des lettres de rémission et que, dans les rares cas où le dénouement de la cause est connue, elle soit soldée par la victoire du ravisseur ou par une simple amende ${ }^{15}$.

Le rapt est avant tout un crime noble. D'après Claude Gauvard, les plaidoiries du Parlement de Paris comptent $29 \%$ de nobles ${ }^{16}$. Dans les procès pour rapt, $64 \%$ des victimes et $87 \%$ des ravisseurs sont qualifiés de noble; exception faite des serviteurs et des prêtres, les complices comme les défenseurs de la victime proviennent également de la noblesse. Si le roi ne légifère pas contre le rapt, c'est peut-être qu'il ne désire pas intervenir dans les stratégies matrimoniales de cette dernière.

Les rapts se répartissent en trois grandes catégories: on peut qualifier la première de rapt d'intérêt. Le mariage qui en résulte apporte au ravisseur l'ascension sociale et l'enrichissement qu'il convoite. Le rapt perpétré pour prévenir un mariage est une

12 De la même façon, Gauvard (1991, p. 244 et 246) a souligné que la distance géographique raréfie le nombre de lettres de rémission demandées dans chaque région: les lettres de rémission concernent davantage les criminels provenant du Nord de la France, particulièrement de la Picardie et de Paris, ce qui ne signifie pas pour autant que la criminalité y soit plus importante. Gauvard remarque en effet que le nombre de lettres de rémission augmente dans le Val de Loire lorsque le roi y déménage sa cour. La fréquence des lettres de rémission, comme les procès, est donc fortement liée à la présence du roi et dans notre cas, du Parlement.

Peut-être le petit nombre de rapts au Parlement de Paris s'explique-t-il par le fait que seuls les cas choquants, exemplaires ou inacceptables, se rendent au Parlement? Pour vérifier cette hypothèse, il faudrait effectuer une comparaison entre les causes du Parlement de Paris et celles des cours seigneuriales ou royales inférieures.

14 Lalement vs Bruneval, Arch. nat., X 2a 17, folio $73 v^{\circ}$.

15 Douze procès rapportent l'existence d'une lettre de rémission. Nous les avons retrouvées dans huit cas seulement. Cependant, seules trois de ces lettres ont fait l'objet d'un entérinement par la cour. Quatre fois, les torts subis par la partie lésée ont été compensés. Le montant des amendes varie de 200 livres tournois (ci-après: l.t.) à 40001 . t., pour un total toujours inférieur à celui demandé par la partie. Nicolas de Bruneval est le seul à être condamné en amende honorable ; mais il a été condamné par contumace au moment de sa fuite. Deux fois, le procureur du roi obtient également une amende profitable; il reçoit la moitié de la compensation attribuée à la partie lésée. 
variation sur le même thème. Il se commet lorsque des membres de la famille en désaccord avec le choix matrimonial d'autres parents, enlèvent la fille pour lui faire épouser un autre candidat. Le dernier type de rapt est issu d'enjeux tout autres. Il s'agit du rapt de séduction, d'un rapt d'amour où deux jeunes gens, face à l'opposition de leurs familles, se voient contraints de fuir et de s'épouser clandestinement.

Un rapt typique met en scène une très jeune fille, orpheline et héritière, riche et noble, enlevée par un écuyer, parfois pauvre, bâtard ou puîné. Celui-ci est toujours assisté de complices recrutés parmi ses serviteurs, ses compagnons, ses amis, ses voisins, ses parents et les parents de sa victime. Enfin, ce sont les amis charnels de la victime qui se portent à sa défense ${ }^{17}$.

Après un enlèvement qui comporte violence et résistance, le rapt suit le cours normal de tout mariage. Les fiançailles sont d'abord nouées, préférablement par un prêtre, puis le mariage est conclu à l'église et suivi de réjouissances. Enfin, le mariage est consommé et la cause s'efface des registres lorsque s'établit la vie conjugale.

Le rapt est un crime, certes, mais dont la déviance est relative et temporaire puisqu'il peut conduire à une situation normale et respectée comprenant vie conjugale et descendance. Il s'agit donc d'une stratégie matrimoniale potentiellement efficace.

Le cas de Marie de Kais, enlevée et épousée en 1406 par Nicolas de Bonneval, est un exemple typique qui a l'avantage d'être très bien documenté ${ }^{18}$. Âgée de sept ans seulement d'après ses «amis charnels», de dix ou onze ans selon le ravisseur, Marie est l'héritière qu'il ne faut pas laisser échapper. Orpheline de père et de mère, riche de 600 à 8001 . t. de rente, elle vit à Révillon en Île-de-France, avec ses tuteurs: sa grand-mère maternelle Mahaut de Pas et le mari de celle-ci, Hue de Bliquy. C'est une proie idéale que Nicolas de Bonneval n'a pas tardé à remarquer. Bien que celuici soit de bon lignage - «il est grant gentilz homs, filz de Nicolas de Bonneval et est descendu de moult notable ligne et de ceulx de Saint Martin le gaillart, de la Heuse, d'Estouteville, de Saincte Venne et de Boissay et tous les plus grans de Caux du costé de son pere et quant est du costé de sa mere, il est du lignage au conte de Saint Pol, du vidame d'Amiens et d'autres»" 19 - écuyer trenchant de Louis, duc d'Orléans, et capitaine d'Oulchy, il a soixante ans et ne présente pas un bon parti pour la famille de Marie: «En septembre derrenièrement passé que Nicolas de Bonneval fist parler du mariage. Mais la fille n'a que sept ans et Bonneval est vieil et pour ce si ne $y$ vouldroient consentir les amis de la damoiselle» ${ }^{20}$.

Bonneval prie le duc d'Orléans d'intervenir en sa faveur mais la famille de Marie, ses tuteurs et son oncle Jehan de Cissy dit Lalement, font la sourde oreille. La persuasion ne fonctionnant pas, Bonneval prend les grands moyens. Il se présente avec plusieurs complices ou envoie le bailli de Coucy - selon les versions - à l'hôtel de Marie pour l'enlever.

Lalement et les autres amis charnels de Marie affirment qu'il y a eu rapt. Bonneval s'est servi de la force pour emmener non seulement la fille, réfugiée dans la cave, mais aussi sa grand-mère et son oncle. Il les a contraints à consentir aux fian-

\footnotetext{
17 Sur les amis charnels, voir Turlan (1969).

18 La cause de Marie de Kais s'étale sur de nombreux registres: Arch. nat., X 2a 14, fol. $375 v^{\circ}$ à X $2 a$ 17, fol. $162 \mathrm{r}^{\circ}, \mathrm{X} \mathrm{2a} \mathrm{15}$, fol. $204 v^{\circ}$ à X 2a 16, fol. $93 \mathrm{v}^{\circ}, \mathrm{JJ} 163$, lettre 291 et JJ 166, lettre 254.

19 Lalement vs Bonneval, Arch. nat, X 2a 14, fol. $379 v^{\circ}$.

20 Lalement vs Bonneval, Arch. nat., X 2a 14, fol. $379 r^{\circ}$.
} 
çailles à Chauconin puis au mariage à Paris, en les menaçant et en les enfermant. La grand-mère, âgée de quatre-vingts ans, est morte quelque temps après du deuil que lui a causé ce mariage. Et, cause ultime du procès, Bonneval s'est alors emparé des héritages de la vieille femme, déclarant qu'ils appartenaient à Marie alors que Lalement se dit héritier de sa mère.

Pour Bonneval, il n'y a eu aucun rapt. Au contraire, la famille de Marie, flattée d'être sollicitée par le duc d'Orléans, a consenti au mariage et a même accompagné Bonneval et Marie jusqu'à Paris pour aller remercier le duc d'Orléans:

Ce pendant s'esbatirent ensemble, dansoient et chantoient et firent les fiancailles du gré et consentement de ladicte Marie, de ladicte Mahaut, de Lalement et de tous les amis qui la estoient (...) fut ledit mariage solemnisé et apres les nopces faictes, lesdiz amis de ladicte Marie mercièrent le dit monseigneur d'Orleans de l'onneur qu'il avoit fait a leur parenté et lui recommande les deux mariez et lors le dit monseigneur d'Orleans leur dist qu'il leur feroit du bien ${ }^{21}$.

Le mariage a été consommé: dorénavant, Bonneval appelle Marie «sa vraye femme couchant avecques luin ${ }^{22}$. Peu de temps après, la grand-mère décède, non pas de courroux, mais de maladie et de vieillesse.

Voici donc une querelle d'héritage à laquelle se mêle un mariage conclu trop tôt selon le droit canon, un mariage sinon fait par force, du moins par persuasion, et qui devient un bon prétexte pour traîner l'adversaire en justice.

C'est en avril-mai 1407 que la cause apparaît pour la première fois devant le Parlement de Paris. Bonneval peut alors compter sur un allié important: le duc d'Orléans. Une lettre de rémission du 19 janvier 1408 révèle que le duc a obtenu que le roi lui-même juge cette cause; une date a été fixée au mois d'août 1407. Malheureusement, Bonneval tombe malade avant cette date et quitte Paris, brisant l'arrêt qui ne l'avait élargi qu'à l'intérieur des murs de la métropole: «par le conseil des phisiciens qui n'y savoient mettre remede que de prendre l'air du pais de sa nascion, y feust alé et avant qu'il ait esté garry y ait longuement demouré.» Il ne se présente pas à Paris au mois d'août et se fait «essoiner» ${ }^{23}$. En août, il est condamné par contumace à payer $4000 \mathrm{l}$. t. d'amende aux parties, $2000 \mathrm{l}$. t. au procureur du roi, à convertir 2001 . t. en messes pour l'âme de la grand-mère et à rendre les biens de la fille et de son oncle; banni du royaume, ses biens sont confisqués. La cour lui ordonne également d'amener Marie pour qu'elle soit séquestrée. La lettre de rémission de janvier 1408 lui remet le bannissement et les amendes, sous condition qu'il amène Marie à la cour du Parlement.

Cette lettre n'est jamais entérinée. Entre 1407 et 1412, peu de traces du procès, exception faite de quelques commissions en 1409 où l'on découvre que Lalement et d'autres amis charnels de Marie tentent de poursuivre le prévôt et des serviteurs de l'évêque de Beauvais et nombre d'autres défendeurs. Puis, en 1412, le procès reprend. Nicolas de Bonneval s'est rendu à la justice après avoir obtenu une autre lettre de rémission datée du mois d'août 1412 où il avoue avoir enlevé Marie de son consentement, ce qu'il ne reconnaissait pas dans la première lettre. Il est désormais prisonnier au Châtelet de Paris et Marie a été remise aux mains du Parlement. Les circonstances

\footnotetext{
$21 \quad$ Lalement $v s$ Bonneval, Arch. nat., X 2a 14, fol. $379 v^{\circ}$ et $X 2 a 17$, fol. $113 r^{\circ}-133 v^{\circ}$.

22 Lalement $v s$ Bonneval, Arch. nat., X 2a 14, fol. $381 v^{\circ}$.

23 Un accusé se fait «essoiners lorsqu'il envoie quelqu'un, un parent ou un ami, déclarer sous serment à la cour qu'il est malade et qu'il n'a pas pu se présenter en cour.
} 
sont différentes: Louis d'Orléans a été assassiné en novembre 1407 et le conflit entre les Armagnacs et les Bourguignons fait rage; Bonneval profite sans doute du répit de la réconciliation d'Auxerre en août 1412 pour reprendre son procès ${ }^{24}$.

Lalement et les autres amis charnels de Marie commencent par refuser l'entérinement des deux lettres de rémission. On apprend ensuite que Bonneval a conclu un accord avec d'autres amis charnels de Marie, accord auquel s'oppose Lalement. Il est alors tout à fait clair que Lalement tente de garder pour soi l'héritage de Marie. La cause traîne encore un an. Bonneval demande sans cesse son élargissement afin d'aller gérer ses biens et de payer l'amende.

Les dernières traces de cette histoire surgissent en 1414, huit ans après le crime. Le mariage de Bonneval et de Marie de Kais qui a maintenant au moins quatorze ans, semble être un fait acquis. Bonneval ne cesse de l'appeler sa femme et il semble qu'une relation de couple se soit installée entre cet écuyer sexagénaire et cette jeune adolescente. En effet, on apprend que Marie a avoué en confession «que Bonneval est son mary et qu'elle le veult avoirs ${ }^{25}$.

La fin du procès est inconnue. Pouvons-nous imaginer que l'accord a enfin été ratifié, que Bonneval a fini par payer l'amende et qu'il est demeuré avec sa femme? C'est du moins l'impression qui reste lorsque la cause disparaît des registres.

Les plaidoiries du procès de Nicolas de Bonneval en disent long sur les conceptions du mariage véhiculées par les individus et leurs avocats. La cause de Marie de Kais et les autres causes de rapt tirent ainsi un portrait du mariage et de son déroulement, d'autant plus que les plaidoiries discutent davantage de la validité du mariage que des caractéristiques criminelles du rapt, en particulier de l'utilisation de la violence.

L'exemple de Marie de Kais démontre que la violence, ou du moins sa mention en cour, se concentre au moment de l'enlèvement. Les demandeurs cherchent systématiquement à prouver l'utilisation de la force lors de l'enlèvement ${ }^{26}$. Voilà qui est conforme au droit: un enlèvement n'est criminel que s'il a été commis contre la volonté de la fille. Ainsi, le juriste Beaumanoir affirme que la victime doit prouver qu'elle a protesté, qu'elle a tenté de s'enfuir ou que son ravisseur a menacé sa vie ou celle de membres de sa famille ${ }^{27}$.

Vingt-trois causes décrivent le déroulement d'un enlèvement ${ }^{28}$. Celui-ci ne se commet jamais à l'improviste. Le ravisseur connaît toujours sa victime et, dans la plupart des cas, a déjà eu des rapports avec elle ou avec sa famille: visites, échanges de paroles ou de cadeaux, demande en mariage, même parfois fiançailles. C'est seulement lorsque sa démarche matrimoniale échoue, lorsqu'il se heurte au refus de la fille ou de sa famille qu'il enlève la fille convoitée.

Le plus fréquemment, la fille est ravie directement de son hôtel. Le ravisseur

24

25

26

27

28

\footnotetext{
Au sujet de la querelle des Armagnacs et des Bourguignons, voir Schnerb (1988).

Lalement $v s$ Bonneval, Arch. nat., X 2a 17, fol. $73 v^{\circ}$.
}

Deux exceptions à cette règle se présentent: 1) lorsqu'il s'agit d'un mariage clandestin et de la fuite des deux tourtereaux, le consentement de la victime est alors évident mais la séduction d'une mineure et non l'enlèvement violent constitue ici le cas criminel ; 2) lorsque les défendenrs admettent qu'un rapt a été commis violemment, en particulier dans une lettre de rémission où ils doivent décrire un semblant de vérité s'ils veulent que la lettre soit entérinée par la partie adverse.

Brundage (1987, p. 470).

L'enlèvement de Marguerite de Chauvirey (Pontalier $v s$ d'Oiselier, Arch. nat., X 2a 14, fol. $250 v^{\circ}$ $287 \mathrm{r}^{\circ}$ ) est passé sous silence. 
n'hésite donc pas à violer la sphère privée et à s'y introduire pour y dérober la fille. Claude Gauvard a démontré que la majorité des crimes aux XIV et $X^{\mathfrak{e}}$ siècles se commettent à l'extérieur, au vu et au su de tous ${ }^{29}$. Mais ces crimes sont surtout des rixes-homicides. Les crimes qui portent atteinte à la propriété, comme le vol, se commettent davantage en secret, dans le privé de la demeure. Ils sont commis par des parents, des amis ou des domestiques qui connaissent suffisamment les lieux et leurs occupants pour pouvoir s'y introduire et accomplir leur délit. Le rapt s'apparente donc au vol: d'une part, l'aide des proches de la fille est centrale à la réussite du crime; d'autre part, le rapt, en tant que vol de la femme et par conséquent de son héritage et de ses biens, est en partie un crime contre la propriété.

Il arrive que l'enlèvement se produise à l'extérieur lorsque la fille se transporte d'un lieu à un autre, de la maison à l'église par exemple. Pendant ces déplacements, la fille n'est jamais d'accès facile. Elle n'est jamais seule, bien gardée, encadrée de parents, de gardiens ou de serviteurs. L'enlèvement de la fille ne résulte jamais de leur négligence.

Cette surveillance explique pourquoi l'enlèvement est une entreprise préméditée et organisée où l'utilisation de la violence est nécessaire. En tout et pour tout, seuls cinq procès ne mentionnent ni force ni menaces, ni armes ni complices au moment de l'enlèvement ${ }^{30}$. Le cas de Marguerite de L'Eglentier témoigne des moyens utilisés par les assaillants:

Pierre de Luilly acompagnié de Robert d'Aucy et de plusieurs autres gens armez de diverses armes (...) vindrent audit lieu de Franceures environ heure de la nuytant apres souleil couché et soupoit lors la dicte damoiselle et appella a l'uys icelui Pierre qui y entra et aussi ledit Robert et plusieurs autres et disoit ledit Pierre que s'il avoit homme qui bougast qu'il seroit mort. Et fu prise la dicte demoiselle par les braz et trainée dehors par ledit Pierre, Robert et autres non obstant qu'elle criast a haulte voix. Et fu par eulx montée sur un cheval derriere ledit Pierre dont elle chey en la boe plusieurs foiz et la remontèrent derechief a che$v a l^{31}$.

Les ravisseurs n'agissent jamais seuls. Le nombre des complices peut varier grandement: trois, dix, douze, vingt complices et même de véritables armées de soixante à cent vingt hommes. Même en admettant que les demandeurs gonflent sciemment le nombre de complices, l'effet est réussi. Pour paraître plus menaçants, pour réussir l'enlèvement et contrecarrer toute opposition, les ravisseurs et leurs complices sont souvent «armez diverses armes» ${ }^{32}$. En alléguant l'emploi d'armes, les demandeurs rendent d'autant plus crédibles les coups et les menaces de mort.

Ces violences ne visent pas uniquement la victime; ses compagnons sont souvent injuriés, parfois même battus. Seuls les demandeurs rapportent ces mauvais traitements car les défendeurs ont intérêt à ce que des accusations pour injure et «bature» ne soient pas additionnées à celle de rapt. Pour se disculper, les défendeurs

29 Gauvard (1991, p. 281-288).

30 Il s'agit de Gobin vs Mes, Arch. nat., X 2a 14, fol. $100 \mathrm{r}^{\circ}$, Pontalier vs d'Oiselier, Arch. nat., X 2a 14, fol. $250 v^{\circ}-287 r^{\circ}$, Boucicaut, Arch. nat., X $2 a$ 17, fol. $134 v^{\circ}-136 v^{\circ}$ et X 2a 16, fol. $238 r^{\circ}-238 v^{\circ}$, Montbrun vs Delroure, Arch. nat., X 2a 24, fol. $21 r^{\circ}-118 r^{\circ}$ et X 2a 23, fol. $149 r^{\circ}-227 r^{\circ}$ et Pronnier $v s$ Rochefort, Arch. nat., X 2a 25, fol. $139 \mathrm{r}^{\circ}-142 \mathrm{v}^{\circ}$.

31 L'Eglentier vs D'Aucy, Arch. nat., X 2a 12, fol. $163 v^{\circ}$.

32 L'Eglentier vs D'Aucy, Arch. nat., X 2a 12, fol. $169 v^{\circ}$. 
nient violence et résistance. La plupart d'entre eux affirment carrément qu'il n'y a eu aucun rapt, à l'instar de Nicolas de Bonneval qui clame: «ny ot ne fut rapt» ${ }^{33}$.

Au moment de l'enlèvement, la réaction de la fille, du moins celle décrite par les demandeurs, est conforme au droit. Tout un rituel, comprenant protestations, cris, pleurs et coups, dicte les gestes de la victime, tels ceux d'Ysabeau Morne qu'enlève Regnault le Fauconnier:

Yceulx complices prindrent Ysabeau la fille (...) et par force elle criant a haulte voix la montèrent sur le cheval et au devant dudit Fauconnier et hastivement eulx tous a cheval l'amenèrent au port de Dernant sur Chier. (...) Dit que audit port, les complices passèrent Chier et tousjours plouroit Ysabeau et esgratinoit Regnault qui la tenoit et se defendoit au mieulx qu'elle povoit et estoit sanz chaperon car il l'avoit si tost amenée que elle l'avoit laissié cheoir en chemin et pour ce qu'elle se demenoit comme courroucié la lièrent d'un mantel ${ }^{34}$.

Les cris et les pleurs dénoncent le crime et sauvent l'honneur de la jeune fille en prouvant son opposition à la capture et au viol. Certaines victimes adoptent d'autres formes de résistance: Marguerite de L'Eglentier refuse de manger; Jehanne Hemery s'évanouit; Marie de Kais se cache ${ }^{35}$. De plus, les parents s'opposent et résistent eux-mêmes à cet enlèvement commis contre leur volonté et contre l'autorité paternelle et familiale.

À huit reprises, demandeurs et défendeurs ne se soucient cependant pas de décrire la réaction de la fille. Plus on avance dans le $X^{e}$ siècle et moins les plaidoiries mettent l'accent sur la résistance de la victime. Les sentiments de la fille deviennent-ils un détail sans importance? Le rituel de résistance perd-il peu à peu de sa signification? Il semble surtout que l'argumentation dans les procès pour rapt se soit déplacée du moment de l'enlèvement à celui du mariage. Un rapt se prouve mieux par l'invalidité d'un mariage obtenu violemment et sans consentement féminin que par la résistance futile d'une jeune et faible fille, sans effet face à des hommes nombreux, violents et armés.

Pour condamner le rapt, les demandeurs insistent donc sur l'illégalité d'un mariage hors normes. C'est ainsi que les procès font ressortir les normes matrimoniales médiévales et les temps forts qui viennent prouver la réalité d'un mariage: les fiançailles, le consentement des époux, le consentement des parents, la consommation, les rites religieux et profanes.

Viennent d'abord les fiançailles. Même si elles ne sont pas essentielles à la validité d'un mariage, même si l'Église ne les impose pas, les fiançailles constituent une étape normale vers la conclusion d'un mariage. Elles le préparent et le contraignent à se réaliser ${ }^{36}$. Elles sont donc mentionnées dans la majorité des causes.

La narration des fiançailles et leur place dans la trame des événements permet de distinguer deux types de rapts: celui où les fiançailles précèdent l'enlèvement et celui où elles lui succèdent. Treize fois, les fiançailles ont été célébrées avant l'enlè-

Lalement $v s$ Bonneval, Arch. nat., X 2a 17, fol. $113 v^{\circ}$.

Morne vs Maleret, Arch. nat., X 2a 14, fol. $224 v^{\circ}$.

L'Eglentier vs D'Aucy, Arch. nat., X 2a 12, fol. $163 v^{\circ}$; Hemery vs D'Asincourt, Arch. nat., X 2a 14, fol. $242 v^{\circ}-243 r^{\circ}$ (Jehanne Aymery, fille de Pierre Aymery, procureur au Parlement); Lalement vs Bonneval, Arch. nat., $X$ 2a 17, fol. $73 \mathrm{r}^{\circ}$.

Sur les fiançailles voir Le Bras (1968, p. 197), Gaudemet, (1987, p. 165-171) et Lefebvre-Teillard (1980, p. 43-49). 
vement. Dans ce cas, l'enlèvement impose simplement la conclusion d'un mariage qui tarde à se nouer. Un changement de circonstances ou d'opinion est peut-être venu interrompre la démarche matrimoniale ${ }^{37}$. En d'autres cas, les parents se sont opposés à des fiançailles clandestines ${ }^{38}$. Ne servant qu'à conclure une démarche matrimoniale déjà amorcée, le rapt n'est pas nécessairement violent et la fille consentante y résiste peu. Par ailleurs, les fiançailles suivent sept fois l'enlèvement. Voilà les rapts violents qui imposent les fiançailles à la fille contre sa volonté et contre celle de ses parents ${ }^{39}$.

Cependant, il ne suffit pas d'imposer les fiançailles ou même le mariage. Pour que celui-ci soit valide, il faut obtenir le consentement de la fille. Le ravisseur et les autres défendeurs, $s$ 'ils espèrent remporter le procès, doivent donc prouver que les fiançailles et le mariage ont été conclus «du bon gré et volenté de la dicte damoiselle» ${ }^{40}$.

L'influence de l'Église est ici manifeste: le droit canon affirme que le consentement des époux est la fondation du mariage. Demandeurs et défendeurs appliquent ce droit dans les plaidoiries et tentent de prouver ou d'infirmer l'existence de ce consentement. Ce sont quinze défendeurs qui avancent l'existence de ce consentement alors qu'onze demandeurs le réfutent et affirment que la fille «ne se vouloit consentir audit mariage» ${ }^{41}$.

Le consentement de la fille est donc pris en compte dans plus de la moitié des causes. Il apparaît nécessaire aux fiançailles et au mariage, non pas qu'il compte réellement mais parce qu'il constitue un argument juridique essentiel au succès du procès. On se rend compte à quel point ce consentement est superficiel lorsque la victime «consentante» traîne son «époux » en cour, comme dans le cas de la veuve Marguerite de L'Eglentier qui intente un procès pour rapt à son soi-disant mari, Pierre de Luilly, et à ses complices ${ }^{42}$. En insistant sur le consentement de la fille, les parties comme les procureurs d'une cour laïque reconnaissent et appliquent pour fins de plaidoiries les règles de la doctrine ecclésiastique.

Cependant, plus que le consentement de la fille, c'est surtout le consentement des parents, le «consensus amicorum», qui compte, même si Robinet de Wastepaste

37 Par exemple, le père de Marguerite de Chauvirey a planifié son mariage avec Gauthier d'Oiselier (Vauthier d'Oiselet). La mort du père est venue interrompre ces projets de mariage. Pontalier vs d'Oiselier, Arch. nat., X 2a 14, fol. $251 \mathrm{v}^{\circ}-252 \mathrm{r}^{\circ}$.

38 L'enlèvement de Jehanne de Cassel est du premier type : de crainte que la famille de Jehanne ne s'oppose à leur mariage, les deux amoureux, déjà fiancés, doivent fuir Lille pour s'épouser (Cassel $v s$ Wastepaste, Arch. nat., X 2a 24, fol. $98 v^{\circ}-207 v^{\circ}$ et X 2a 23, fol. $194 v^{\circ}-351 v^{\circ}$.) Nicaise le Caron enlève Margot de Notre-Dame pour conclure le mariage planifié par la belle-mère de Margot, scur de Nicaise, contre la volonté de certains autres membres de la famille de Margot. Les fiançailles ont déjà eu lieu. (Hardencourt vs Caron, Arch. nat., JJ 118, fol. 183 v $^{\circ}$ ).

39 Pourtant, même dans ces circonstances, l'emploi de la violence est rare: seulement cinq filles sont menacées, rudoyées ou effrayées au moment des fiançailles, et quatre d'entre elles à nouveau au moment du mariage. Voilà qui vient confirmer le fait que la violence est surtout nécessaire au moment de l'enlèvement.

40 Warisonne vs Bezon, Arch. nat., JJ 121, lettre 216, fol. $115 \mathrm{v}^{\circ}$.

41 Eschalart vs Aubigne, Arch. nat., X 2a 18, fol. $243 \mathrm{v}^{\circ}$. Sur l'importance du consentement des époux, voir Sheehan (1978), Noonan (1973), Donahue (1976) et (1983).

42 L'Eglentier vs D'Aucy, Arch. nat., X 2a 12, fol. $163 \mathrm{v}^{\circ}-211 \mathrm{v}^{\circ}$, X 2a 11, fol. $156 \mathrm{v}^{\circ}$, X 2a 13, fol. 118 $v^{\circ}-124 r^{\circ}, J J 143$, fol. $39 v^{\circ}-40 r^{\circ}$ et fol. $83 v^{\circ}-84 r^{\circ}$. 
affirme que «licet de honestate l'en doye appeler les parens et amis toutefuoies ce $n^{\prime}$ est pas de necessitate» ${ }^{43}$.

Les défendeurs sont conscients de l'importance de ce consentement parental. Voilà pourquoi ils déclarent fréquemment que les parents ont donné leur consentement ou du moins qu'ils ne se sont pas opposés ouvertement: «n'y ot parent de la fille ne autre qui y mist empeschement $\rangle^{44}$. Les chiffres sont éloquents: fiançailles et épousailles conjuguées, l'avis des parents est pris en compte par dix-sept défendeurs. Les parents doivent être consultés. Ignorer ou s'opposer à la volonté des parents est une offense qui vient aggraver la cause du ravisseur.

Les demandeurs, parents et amis des victimes, accordent presque autant d'importance à leur approbation. Treize d'entre eux déclarent s'être opposés aux fiançailles ou au mariage: «ne y vouldroient consentir les amis de la demoiselle» ${ }^{45}$. Voilà l'argument le plus utilisé par les demandeurs pour prouver que le mariage contracté par rapt est, sinon invalide, du moins mauvais. C'est aussi celui qu'utilisent les procureurs du roi, par exemple contre Hutin de Clamas, ravisseur de Perrotine des Sarteaulx: «Pose que les filles feussent d'accord a aucuns se les parens et amis veoient ce inhonestim vel inutile elles estoient reputées non consentante. Car aussi le consentement des amis par raison civile y est necessaire» ${ }^{46}$.

C'est sûrement l'argument qui vise le plus juste. Les parents représentent l' autorité au sein de la famille, celle qu' on ne peut bafouer. Dans une société où la famille est le noyau de base, où les enfants doivent obéissance à leurs parents, où les parents ont tout pouvoir décisionnel, il paraît tout-à-fait normal que les parents soient ceux qui décident du mariage, ceux qu'on consulte obligatoirement, malgré le droit canon et les discours des ecclésiastiques qui voudraient laisser le libre choix aux époux ${ }^{47}$. On touche certainement ici au cœur du conflit qui entoure de nombreux rapts.

Quelques défendeurs admettent toutefois le refus des parents. Dans ces rares cas, c'est l'opposition parentale qui justifie le rapt. La fille a donné son accord mais le couple amoureux se heurte au refus parental. Le rapt devient alors un rapt de séduction: le couple avait-il d'autre choix que de s'enfuir pour faire triompher son amour dans l'honnêteté du mariage? Jehan Gobert l'exprime clairement:
Que comme Jehan Gobert escuier (...) et Katherine la Prevoste (...) qui avoient grant amour ensemble en esperance de prendre l'un l'autre et estre conjoins par mariage et doubtoient que les amis de la damoiselle ne fussent pas bien en accord dudit mariage, feussent en accort que le dit escuier et autres de ses amis en sa compaingnie vendroient en certain lieu pres de la dicte ville de Tournay et que la vendroient aussi la dicte damoiselle et sa mere. Et lors icellui escuier par lui et par ses amis dessus dis prendroit de fait la dicte damoiselle et la meneroit la ou bon li sembleroit pour la espouser ${ }^{48}$.

Mais que vaut l'amour et la volonté personnelle devant la puissance des parents et de la famille? Jehan et Katherine, chassés de leur ville natale et réduits à demander une lettre de rémission dans l'espoir de parvenir à s'épouser, l'ont appris à leurs dépens.

\footnotetext{
43 Cassel vs Wastepaste, Arch. nat., X 2a 24, fol. $105 \mathrm{r}^{\circ}$.

44 Cassel vs Wastepaste, Arch. nat., X 2a 24, fol. $102 v^{\circ}$

45 Lalement vs Bonneval, Arch. nat., X 2a 14, fol. $379 \mathrm{r}^{\circ}$

46 Paris vs Clamas, Arch. nat., X 2a 14, fol. $106 \mathrm{r}^{\circ}$.

47

Au sujet de l'autorité parentale, voir Vantroys (1899), Finch (1990) et la réponse de Donahue (1992).

48

Warisonne vs Bezon, Arch. nat., JJ 121, lettre 216, fol. $115 \mathrm{v}^{\circ}$.
} 
La question du consentement - celui de la fille à cause du droit, celui des parents à cause de la tradition - occupe tout l'avant-scène de la discussion entourant la validité d'un mariage. Cependant, d'autres facteurs viennent aussi sinon valider le mariage, du moins prouver qu'il s'est bel et bien noué.

On pourrait croire que la consommation occupe un rôle important comme acte fondateur du mariage et comme preuve de son existence. Les enfants ne sont-ils pas le but premier d'un mariage? La nuit de noces n'occupe-t-elle pas une place centrale dans le rite du mariage, venant ratifier l'union de l'homme et de la femme? Même les théologiens ont longtemps tergiversé pour savoir quelle valeur attribuer aux premières relations sexuelles dans la formation du mariage. Ils ont finalement opté pour le consentement comme acte fondateur de l'union matrimoniale, reléguant la consommation au deuxième rang ${ }^{49}$. Nos documents abondent dans le même sens: ils révèlent que le consentement, du moins comme argument juridique, sert de première preuve à la validité d'un mariage et que la consommation n'est qu'une preuve supplémentaire et accessoire.

En fait, l'attitude des parties envers la consommation est ambivalente. D'une part, les relations sexuelles semblent ratifier le mariage, le sceller et le rendre indissoluble. Ainsi, à neuf reprises, les défendeurs allèguent «le mariage fait et consommé»" ${ }^{50}$. Parfois même, ils affirment que la fille est "grosse» ou qu'elle a eu des enfants: «a esté consommé le mariage telement qu'elle en a deux enfans a une ventrée» ${ }^{51}$. Ils cherchent ainsi à prouver la réalité du mariage, et avec les enfants, sa valeur.

D'autre part, les relations sexuelles ne semblent nullement essentielles à la création d'un mariage valide. Elles peuvent même constituer une circonstance aggravante. Après que Nicolas de Bonneval ait annoncé la consommation de son mariage avec Marie de Kais, le procureur du roi répond «que attendue la presumpcion de Bonneval qui dit que le mariage est consummé per copulam carnalem le cas $s^{\prime}$ agrave ${ }^{2}$. Certes, le très jeune âge de Marie peut expliquer la réaction du procureur du roi. Mais sept autres demandeurs mentionnent aussi en termes accusatoires la consommation du mariage et la corruption de la fille. Règle générale, les demandeurs préferent rapporter la virginité intacte de la victime, preuve que le mariage n'a pas été consommé et qu'il peut encore être dissous et preuve qui, surtout, rétablit l'honneur froissé de la victime.

De plus, et cela prouve le peu d'importance attribué aux relations sexuelles, le fait que le mariage ait été consommé n'empêche pas les demandeurs d'exiger la dissolution du mariage - «qu'ilz rendent ladite fille franche et desliée de tout lien de

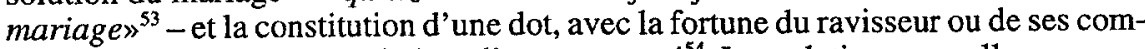
plices, afin de pourvoir la victime d'un autre mari ${ }^{54}$. Les relations sexuelles ne compromettent donc pas tout projet futur de mariage.

49 Sur le rôle de la consommation dans la doctrine ecclésiastique, voir Noonan (1973), LefebvreTeillard (1980, p. 47-49) et Gaudemet, (1987, p. 175-176).

50 Pontalier vs d'Oiselier, Arch. nat., X 2a 14, fol. $252 \mathrm{r}^{\circ}$.

51 Merle $v s$ Bertrand, Arch. nat., X 2a 24, fol. $194 \mathrm{v}^{\circ}$.

52 Lalement vs Bonneval, Arch. nat., X 2a 14, fol. $381 \mathrm{v}^{\circ}$.

53 Pontalier vs d'Oiselier, Arch. nat., X 2a 14, fol. $250 v^{\circ}$.

54 «que icelluy d'Aubigne soit condemné et contraint a luy bailler et asseoir bien et convenablement IIT livres de rente apperpetuité afin de mieulx et plus honnorablement elle puisse estre marié» (Eschalart vs Aubigne, Arch. nat., X 2a 18, fol. $244 \mathrm{v}^{\circ}$ ). 
Les relations sexuelles suivant un rapt ne sont jamais qualifiées de viol. Il est même rare qu'on mentionne la réaction de la fille, sa résistance ou l'emploi de la force. Cette réaction importe peu car un mariage et non un viol est au cœur du procès. Or, une femme mariée ne peut pas être violée par son mari ${ }^{55}$. Les demandeurs doivent surtout prouver l'invalidité du mariage; la discussion entourant les relations sexuelles n'apporte rien à leur plaidoirie. Pour les défendeurs, la preuve d'une consommation peut démontrer la réalité du mariage, mais peut également amener méfiance et désapprobation.

Les relations sexuelles sont sûrement importantes dans un mariage, considérées comme une suite normale à l'échange des consentements, mais elles ne sont pas la cause du procès et elles y ont laissé somme toute peu de traces. Pour la consommation comme pour le consentement, les parties et les procureurs au Parlement adoptent les règles ecclésiastiques quand vient le temps de plaider et de juger un mariage.

Le respect des rites matrimoniaux traditionnels et surtout des rites religieux est un argument plus utile pour prouver l'existence d'un mariage. Tout mariage se célèbre à l'église: treize fois, les demandeurs ou les défendeurs affirment que le mariage a été fait «en face de saincte eglise». Lorsque le mariage n'a pas été célébré religieusement, ce sont les demandeurs qui le dénoncent pour inculper le ravisseur. Ainsi, ils affirment que Robinet de Wastepaste et Jehanne de Cassel «ne se deussent estre espousez sans messe comme firents ${ }^{56}$.

Un prêtre doit également être présent: il l'est à neuf reprises pour les fiançailles et dix fois pour le mariage. Deux fois, on mentionne spécifiquement son absence aux fiançailles, ce qui confirme que des fiançailles normales sont bénies par un prêtre. La preuve: Jehanne de Cassel et Robinet de Wastepaste trouvent nécessaire de faire confirmer leurs fiançailles clandestines par un prêtre: «s'en alèrent au curé de Sainte Katherine lequel informé que estoient fiancez par paroles de futur les fianca par paroles de presents ${ }^{57}$.

Tout mariage qui veut être reconnu doit être marqué du sceau ecclésiastique. Si l'on conjugue les renseignements fournis par les fiançailles et par les épousailles, une présence religieuse, que ce soit celle du prêtre ou celle de l'église, n'est absente que dans trois des vingt rapts s'étant conclus par un mariage. Le rituel ecclésiastique a réellement sa place dans la formation des unions matrimoniales et sert d'argument juridique pour en prouver l'existence, la valeur et la validité. L'Église a réussi à s'intégrer dans le tissu des rites matrimoniaux. Si cette intégration n'est pas totale, si certains mariages continuent à se conclure clandestinement, sans aucune présence religieuse, la norme est bien celle d'un mariage in facie ecclesiae.

Au moment de conclure les fiançailles ou le mariage, il arrive que des empêchements se présentent et mettent en péril la validité du mariage ${ }^{58}$. Les demandeurs semblent bien connaître ces empêchements, surtout d'ordre ecclésiastique, et s'en servent pour miner le mariage, preuve que le droit canon est connu et appliqué lorsqu'il peut s'avérer utile.

ss Brundage (1987, p. 396 et 471).

56 Cassel vs Wastepaste, Arch. nat., X 2a 24, fol. $103 v^{\circ}$. Sur les rites matrimoniaux ecclésiastiques, voir en particulier Molin et Mutembe (1974).

57 Cassel vs Wastepaste, Arch. nat., X 2a 24, fol. $102 v^{\circ}$.

58 Sur les différents empêchements et autres causes de nullité prévus par le droit canon, voir Gaudemet (1987, p. 184-185, 197-215). 
Six demandeurs affirment que «le mariage est nul attendu l'aage» ${ }^{59}$ : en effet, bon nombre des victimes ont tout juste douze ans, l'âge canonique minimum pour le mariage des filles. L'inexactitude des âges permet aux demandeurs de jouer cette carte. Les défendeurs ne peuvent que proposer un âge plus élevé pour la jeune fille. L'empêchement est parfois de l'ordre de la consanguinité, mais les défendeurs allèguent toujours une dispense ecclésiastique ${ }^{60}$. Autre argument surtout utilisé au moment des fiançailles, les demandeurs affirment quatre fois que la fille est déjà fiancée ou mariée.

Enfin, un dernier empêchement ne dépend pas du droit canon. La cour du Parlement ou une autre juridiction - par exemple l'évêque de Poitiers ${ }^{61}$ - a parfois interdit le mariage, à cause de disputes familiales ou de l'enlèvement; le ravisseur a fait fi de cette interdiction et a conclu un mariage illégal ${ }^{62}$.

C'est justement pour détecter de tels empêchements et pour garantir la publicité du mariage que le Concile de Latran IV de 1215 a imposé les bans. Ceux-ci, que nos documents appellent «les bans et solemnitez ordennees en sacrement de mariage», semblent cependant moins essentiels que la présence à l'église. Même si leur absence rend le mariage illicite, elle ne l'invalide pas. La partie adverse de Marguerite de L'Eglentier affirme même que «n'estoit necessaire de fere bans ${ }^{63}$. II est donc facile de s'en passer. Dans le cas de rapts, le temps presse et n'offre pas le luxe d'attendre quelques semaines la publication des bans, d'autant plus que ces bans risquent de révéler des oppositions. Par conséquent, les bans n'ont été publiés, selon les défendeurs, que pour cinq mariages.

Si ces bans ne sont pas obligatoires, les défendeurs y font référence pour prouver que le mariage a été fait publiquement, sans que personne ne s'y oppose. Les défendeurs dans le procès pour le rapt de Marguerite de Chauvirey l'affirment: «si furent faictes les fiancailles puis les espousailles liberalement, publicquement, de die et solennelement au moustier parrochial de Bonnencontre, toutes sollenitez y furent gardées ${ }^{64}$. Inversement, les demandeurs démontrent la clandestinité d'un mariage sans bans, dans la peur de la réprobation et du refus familial. Les bans, dans ces plaidoiries, remplissent exactement le rôle que leur a assigné l'Église: publicité et approbation. S'ils sont parfois mentionnés dans ces mariages conclus rapidement, un peu clandestinement, toujours contre certains membres de la famille, imaginons à quel point ils devaient être employés dans le cadre de mariages planifiés et approuvés.

Face aux rites religieux, fiançailles, messe et bans, les rites profanes semblent occuper une place marginale dans la célébration du mariage puisqu'ils ne sont men-

59 Lalement vs Bonneval, Arch. nat., X 2a 14, fol. $381 \mathrm{r}^{\circ}$. Cette objection est toujours présentée au moment du mariage; en effet, les fiançailles sont possibles à partir de l'âge de sept ans et toutes les victimes ont au moins sept ans. Sur l'âge au mariage, voir Onclin (1965).

so Dans le cas des fiançailles de Jehanne Jourdaine et de Guillaume Josseaume (Lestang vs Josseaume, Arch. nat., X 2a 17, fol. $123 \mathrm{v}^{\circ}-124 \mathrm{r}^{\circ}$ ) et du mariage d'Anthoinete de Cravant et de Joceaume Bertrand (Merle vs Cravant, Arch. nat., X 2a 24, fol. $195 v^{\circ}$ ).

61 Eschalart vs Aubigne, Arch. nat., X 2a 18, fol. $243 v^{\circ}$.

62 Par exemple, la cour a défendu que le mariage de Marguerite de Chauvirey ne soit traité sans son accord et celui des amis à peine de 1000 marcs d'argent d'amende, mais Gauthier d'Oiselier (Vauthier d'Oiselet) l'a épousée tout de même (Pontalier vs d'Oiselier, Arch. nat. X 2a 14, fol. $250 \mathrm{v}^{\circ}$ ). L'Eglentier vs D'Aucy, Arch. nat., X 2a 12, fol. $166 \mathrm{r}^{\circ}$. Sur les formalités entourant le mariage, voir Le Bras (1927, p. 198-199) et Gaudemet, $(1987$, p. 231). 
tionnés que trois fois. Cela ne signifie pas que les réjouissances n'ont pas leur place dans le déroulement d'un mariage noble, mais plutôt que les circonstances d'un rapt et d'un mariage clandestin se prêtent mal aux célébrations et aux grandes réunions.

Lorsqu'elles sont mentionnées, les célébrations servent à la publicité du mariage et prouvent que la fille était joyeuse et consentante: «Y ot grans foison de gens d'onneur aux noces qui furent grans et belles. Et y avoit des meneteres et danca plusieurs foiz la dicte damoiselle» ${ }^{65}$. Après le mariage d'Ysabeau Morne, le ravisseur et ses complices trouvent important de fêter et de manger, preuve qu'un repas fait partie des rites matrimoniaux, quitte à l'imposer de force: «Si fait tuer Maleret trois chapons dudit moine et voult que Ysabeau but et mengast mais elle est triste et dolent nen a cure. Se print Regnault sa dague et a force d'icelle lui ouvri sa bouche et fist geter du vin dedens ${ }^{66}$. Fait intéressant, on arrête de mentionner la présence de réjouissances après $1414^{67}$. On ne saurait croire qu'elles disparaissent des rites matrimoniaux. Les noces perdent-elles alors leur valeur comme preuve juridique de l'existence et de la valeur d'un mariage?

Le contrat de mariage joue le même rôle que les bans et que les noces en tant que garant de la publicité et de l'approbation du mariage, tout en répondant à des préoccupations économiques absentes des formalités religieuses et des réjouissances.

Il va sans dire que les rapts et leurs procès sont chargés d'enjeux économiques: le conflit d'un rapt résulte souvent d'une tentative d'enrichissement, de l'enlèvement d'une héritière dont l'un des plus grands atouts est d'être riche. Les demandeurs eux-mêmes ne sont pas désintéressés: ils cherchent à conserver l'héritage dans leur famille ou le destinent à leurs alliés. De plus, ils espèrent une grosse compensation pour les dommages encourus. De fait, les peines imposées aux ravisseurs sont presque uniquement des amendes monétaires.

Cependant, les enjeux économiques d'un rapt ne sont pas de ceux qui se négocient; le rapt évolue dans un certain désordre qui s'accommode mal d'un geste posé et réfléchi comme un contrat de mariage. Il est donc étonnant de trouver cinq références à des «traités» ou des «instruments». Peut-être l'habitude de consigner par écrit les termes d'un mariage est déjà à ce point ancrée dans les mœurs de la noblesse qu'un mariage sérieux doit obligatoirement s'y conformer?

En fait, de nombreux contrats sont signés au moment des fiançailles. Ils annoncent un mariage; étant donné le nombre de fiançailles conclues avant l'enlèvement, on comprend mieux qu'un contrat de mariage puisse coexister avec la violence d'un rapt. Quoi qu'il en soit, le contrat de mariage semble faire partie du rituel matrimonial et sa mention est une façon de plus d'assurer la régularité du mariage ${ }^{68}$.

La présence d'un anneau sert également de preuve tangible. Symbole échangé au moment des fiançailles ou des épousailles, il témoigne de l'existence d'un lien entre les conjoints. Voilà pourquoi les parties rapportent parfois que le ravisseur «l'espousa d'un annel d'argents ${ }^{69}$.

65 L'Eglentier vs D'Aucy, Arch. nat., X 2a 12, fol. $164 v^{\circ}$.

os Morne vs Maleret, Arch. nat., X 2a 14, fol. $225 \mathrm{r}^{\circ}$.

67 Il pourrait évidemment s'agir d'une aberration statistique, étant donné le petit nombre de causes prises en compte ici.

68 Les contrats de mariage ont beaucoup servi à l'étude du mariage. Voir entre autres Erickson (1990), Lafon (1972) et Mestayer (1979).

69 Paris vs Clamas, Arch. nat., X 2a 14, fol. $106 r^{\circ}$. Sur le rite de l'anneau, voir Molin et Mutembe, (1974, p. 159-178) et Klapisch-Zuber (1979). 
Enfin, la pérennité du mariage est prouvée par l'existence d'une vie conjugale, dernier sceau apposé à l'union matrimoniale. Sept défendeurs, à l'instar de Marie de Kais et Nicolas de Bonneval qui ont «demouré ensemble en lostel du pere dudit suppliant paisiblement et amoureusement comme mary et femme doivent demourer ensemble» ${ }^{70}$, arguent de l'existence d'une vie commune. Cette constatation offre aussi une dernière occasion aux défendeurs d'affirmer les sentiments positifs de la fille à l'égard de son mari. Perrotine des Sarteaulx «estoit courroucié quant Hustin estoit absent»; Katherine de Montbrun affirme que «elle estait contente du mariage»; Jehanne de Cassel, enfin «dist que Robinet estoit son mary et n'en vouldrait point d'autre» ${ }^{71}$. Les défendeurs mettent fin à leur plaidoirie avec une impression de mariage réussi et de couple heureux.

Dans l'argumentation des plaidoiries au Parlement de Paris, la violence n'est pas la pierre d'achoppement du rapt. Est-ce dû au peu d'efficacité de la résistance de la femme ? Ou est-ce parce que le Parlement de Paris, une fois son autorité sur les rapts admise, a de moins en moins besoin d'insister sur la violence de ceux-ci ? La juridiction du roi acceptée et justifiée, les procureurs et les avocats du Parlement de Paris peuvent utiliser d'autres arguments et surtout discuter de la validité du mariage, même si cette question relève théoriquement des cours ecclésiastiques. Il est d'ailleurs possible que certaines causes soient déjà passées devant une cour ecclésiastique ou y passent parallèlement au procès au Parlement.

Par conséquent, les plaidoiries traitent surtout du mariage et de sa validité. Il en ressort que le Parlement, ses avocats et ses procureurs, de même que ses clients surtout des nobles dans le cas de rapts - connaissent parfaitement les règles qui valident ou invalident un mariage. Ils sont capables de les appliquer pour innocenter ou pour attaquer le ravisseur. Ces règles sont celles qu'a imposées l'Église: le consentement des époux et la publicité du mariage à travers une série de rites religieux. Les propos des parties démontrent également que la consommation et les rites profanes ne jouent qu'un rôle secondaire, du moins comme preuve du mariage. Enfin, ils démontrent surtout l'importance primordiale du consentement parental.

Jusqu'à quel point les plaidoiries reflètent-elles la réalité du mariage? Il ne fait aucun doute que les parties se servent des divers éléments du mariage comme autant d'outils pour prouver leur cause. Elles appliquent le seul droit matrimonial, le droit ecclésiastique. Ce faisant, elles déforment les faits et mettent l'accent sur les arguments qui jouent à leur avantage.

Il semble cependant que les rites religieux aient été bien intégrés aux célébrations entourant un mariage. Sinon, pourquoi les parties insisteraient-elles tant sur les fiançailles, la présence d'un prêtre, le mariage in facie ecclesiae et la publication des bans? Ces rites semblent faire partie d'un mariage normal.

Par contre, les plaidoiries ne révèlent pas tout le rôle des célébrations profanes qui occupent probablement une place toute aussi importante que les rites religieux. Le fait qu'un contrat, un repas, une danse ou des invités soient mentionnés dans ces cas très peu propices aux célébrations prouve leur importance. Mais ces éléments ne constituent pas des preuves très utiles.

70 Lalement $v s$ Bonneval, Arch. nat., JJ 166, lettre 254, fol. $168 \mathrm{r}^{\circ}$.

71 Paris vs Clamas, Arch. nat., X 2a 14, fol. $106 v^{\circ}$; Montbrun vs Delroure, Arch. nat., X 2a 24, fol. 49 $v^{\circ}$; Cassel vs Wastepaste, Arch. nat., X 2a 24, fol. $103 \mathrm{r}^{\circ}$. 
La place de la consommation est moins facile à cerner: elle semble venir confirmer un mariage, surtout par la production d'enfants, mais les populations civiles comme les autorités laïques ne conferent pas à l'acte charnel un rôle-clé dans la fondation du mariage. Ici, modèle ecclésiastique et modèle läque semblent donc s'aligner, l'un et l'autre reconnaissant la place de la sexualité dans le mariage, mais ni l'un ni l'autre ne lui attribuant un rôle fondateur.

Quant à la règle ecclésiastique principale, celle dictant que le consentement des époux forme le mariage, il est difficile de déterminer son poids réel. Il ne fait pas de doute que cette règle est connue de tous. Mais les discussions entourant le consentement de la fille, tout comme les descriptions de l'enlèvement et du mariage, viennent surtout prouver à quel point ce consentement est accessoire. Puisque l'Église le requiert, on avance qu'il existe. Mais comment croire en sa réalité lorsque la fille est menacée et violentée ou lorsque les parents font tout en leur pouvoir pour contrer son choix matrimonial? En fait, le consentement des époux et particulièrement le consentement féminin demeurent difficiles à accepter, surtout pour des parents qui veulent absolument être consultés.

Car il est un élément non-ecclésiastique que les plaidoiries ne minimisent certainement pas: le rôle des parents et de leur consentement. Ce sacro-saint consentement, l'argument le plus utilisé, constitue non seulement le point de départ mais également la base de tout mariage. Il est à la source du conflit de la plupart des rapts, et également du conflit entre l'idéal religieux et les normes laïques.

L'analyse des arguments des plaidoiries pour rapt prouve que, malgré des siècles de campagne ecclésiastique, un modèle aristocratique de mariage demeure profondément ancré dans la pratique. Certes, la lutte n'est plus aussi ouverte qu'au haut Moyen Age ou qu'au XI ${ }^{e}$ siècle décrit par Georges Duby. L'Église a réussi à imposer l'interdiction des mariages incestueux - les seules mentions dans nos plaidoiries démontrent que les règles de l'Église sont appliquées, pour condamner un mariage entre cousins ou pour demander une dispense. Elle a également imposé la monogamie et l'indissolubilité du mariage, sans toutefois éliminer les concubinages, la bigamie et les nombreuses naissances illégitimes. Cependant, la clé de voûte de sa doctrine, le consentement des époux, n'est pas réellement appliquée.

Le modèle matrimonial aristocratique décrit dans les plaidoiries ne semble avoir intégré que les éléments superficiels de la doctrine ecclésiastique et semble s'être détourné du plus essentiel de cette doctrine, du consentement des époux. Le mariage est devenu un amalgame de règles ecclésiastiques et de coutumes laïques, amalgame où l'enveloppe est ecclésiastique - les rites - mais où les motivations profondes demeurent profanes - alliances politiques et économiques imposées par les parents aux enfants.

Cette lutte entre un modèle ecclésiastique et un modèle aristocratique, surtout entre le consentement des enfants et celui des parents, n'est toujours pas résolue à la fin du Moyen Age. En fait, le débat réapparaît de plus belle au XVI ${ }^{\mathrm{e}}$ siècle. L'édit d'Henri II de 1556 rend obligatoire le consentement des parents pour les hommes de moins de trente ans et les filles de moins de vingt-cinq ans. De son côté, le décret Tametsi du Concile de Trente vient timidement appuyer cette mesure du roi de France en proclamant l'horreur de la Sainte Église pour les unions conclues sans le consentement des parents et en imposant la publication des bans et la célébration du mariage in facie ecclesiae ${ }^{72}$. L'importance du consentement des parents est ainsi 
reconnue par les autorités ecclésiastiques et civiles, légitimant du coup les enjeux socio-politiques et économiques des stratégies matrimoniales de la noblesse.

Geneviève Ribordy

455 rue Lemesurier

Québec, QC, GIS IP8

Canada

(Rcharest@compuserve.com)

\section{SOURCES MANUSCRITES}

Archives nationales:

En totalité: X 2a 10, 12, 14, 17, 18, 22, 24 et 25

(Plaidoiries criminelles du Parlement de Paris)

En partie: X 2a 9, 11, 13, 15, 16, 19, 20, 23 et 26

(Lettres et arrêts criminels du Parlement de Paris)

En partie: JJ 118 à JJ 177

(Lettres de rémission conservées au Registre du Trésor des chartes)

\section{RÉFÉRENCES}

Bennett, J., Medieval Peasant Marriage: An Examination of Marriage Licence Fines in the Liber Gersumarum, in Raftis, J.A., (ed.), Pathways to Medieval Peasants, Toronto, Pontifical Institute of Mediaeval Studies, 1981, p. 193-246.

Benveniste, H., Les enlèvements: stratégies matrimoniales, discours juridique et discours politique en France à la fin du Moyen Age, Revue historique, 1990, 283, 573, p. 13-35.

Brundage, J., Law, Sex and Christian Society in Medieval Europe, Chicago, The University of Chicago Press, 1987.

Donahue, C. Jr., The Policy of Alexander the Third's Consent Theory of Marriage, in Kuttner, S., (ed.), Proceedings from the Fourth International Congress of Medieval Canon Law, Vatican, Biblioteca Apostolica Vaticana, 1976, p. 251-281.

Donahue, C. Jr., The Canon Law on the Formation of Marriage and Social Practice in the Later Middle Ages, Joumal of Family History, 1983, 8, 2, p. 144-158.

Donahue, C. Jr., «Clandestine» Marriage in the Later Middle Ages: a Reply, Law and History Review, $1992,10,2$, p. 315-322.

Duby, G., Medieval Marriage. Two Models from Twelfth Century France, Baltimore, John Hopkins University Press, 1978.

Duby, G., Le Chevalier, la femme et le prêtre. Le mariage dans la France féodale, Paris, Hachette, 1981.

Dufresne, J.-L., Les comportements amoureux d'après le registre de l'officialité de Cerisy, Bulletin philologique et historique du comité des travaux historiques et scientifiques, 1976, p. 131-156.

Erickson, A. L., Common Law Versus Common Practice: the Use of Marriage Settlements in Early Modern England, Economic History Review, 1990, 43, 1, p. 21-39.

Esmein, A., Le mariage en droit canonique, Paris, Larose et Forcel, 1891.

Finch, A., Parental Authority and the Problem of Clandestine Marriage in the Later Middle Ages, Law and History Review, 1990, 8, 2, p. 189-204. 
Finch, A., Women and Violence in the Later Middle Ages: the Evidence of the Officiality of Cerisy, Continuity and Change, 1992, 7, 1, p. 23-45.

Gaudemet, J., Sociétés et mariage, Strasbourg, Cerdic, 1980.

Gaudemet, J., Le mariage en Occident. Les mours et le droit, Paris, Cerf, 1987.

Gauvard, C., «De grace especial »: crime et société en France à la fin du Moyen Age, Paris, Publications de la Sorbonne, 1991.

Gottlieb, B., The Meaning of Clandestine Marriage, in Wheaton, R., Hareven, T.K., (eds.), Family and Sexuality in French History, Philadelphia, University of Pennsylvania, 1980, p. 49-83.

Greilsammer, M., Rapts de séduction et rapts violents en Flandre et en Brabant à la fin du Moyen Age, Tijdschrift voor Rechtsgeschiedenis, 1988, 56, p. 49-84.

Greilsammer, M., Les familles en guerre contre la doctrine consensualiste, in L'envers $d u$ tableau. Mariage et maternité en Flandre médiévale, Paris, Armand Colin, 1990, p. 6585.

Helmholz, R. H., Marriage Litigation in Medieval England, Cambridge, Cambridge University Press, 1974.

Ingram, M., Spousals Litigation in the English Ecclesiastical Courts, c. 1350-c. 1640, in Outhwaite, R. B., (ed.), Marriage and Society: Studies in the Social History of Marriage, Londres, Europa Publications, 1981, p. 35-57.

Ingram, M., Church Courts, Sex and Marriage in England, 1570-1640, Cambridge, Cambridge University Press, 1987.

Klapisch-Zuber, C., Zacharie, ou le père évincé. Les rites nuptiaux toscans entre Giotto et le concile de Trente, Annales. Economies, Sociétés, Civilisations, 1979, 34, 6, p. 1216-1243.

Lafon, J., Les époux bordelais. Régimes matrimoniaux et mutations sociales 1450-1550, Paris, S.E.V. P.E.N., 1972.

Le Bras, G., Mariage: la doctrine du mariage chez les théologiens et les canonistes depuis l'an mille, Dictionnaire de théologie catholique, 1927, 9, 2, col. 2123-2223.

Le Bras, G., Le mariage dans la théologie et le droit de l'Église du XI ${ }^{e}$ au XIII ${ }^{e}$ siècle, Cahiers de civilisation médiévale, 1968, 11, p. 191-202.

Lefebvre-Teillard, A., Les officialités à la veille du Concile de Trente, Paris, Libraire générale de droit et de jurisprudence, 1973.

Lefebvre-Teillard, A., Règle et réalité dans le droit matrimonial à la fin du Moyen Age, Revue de droit canonique, 1980, 30, p. 41-54.

Lévy, J.-P., L'officialité de Paris et les questions familiales à la fin du XIV' siècle, in Études d'histoire du droit canonique dédiées à Gabriel Le Bras, Paris, Sirey, 1965, 2, p. 12651294.

Mestayer, M., Les contrats de mariage à Douai du XIII ${ }^{e}$ au XV' siècle, reflets du droit et de la vie d'une société urbaine, Revue du Nord, 1979, 61, 241, p. 353-380.

Molin, J.-B., Mutembe, P., Le rituel du mariage en France du XII au XVI siècle, Paris, Éditions Beauchesne, 1974.

Naz, R., Rapt, Dictionnaire de droit canonique, 1965, 7, col. 454.

Noonan, J. T., Marriage in the Middle Ages: Power to Choose, Viator. Renaissance and Medieval Studies, 1973, 4, p. 419-434.

Onclin, W., L'âge requis pour le mariage dans la doctrine canonique médiévale, in Kuttner, S., Ryan, J. J., (eds.), Proceedings of the Second International Congress of Medieval Canon Law, Vatican, 1965, p. 237-247.

Porteau-Bitker, A., La justice laïque et le viol au Moyen Age, Revue historique de droit français et étranger, 1988, 4, p. 491-526. 
Schnerb, B., Les Armagnacs et les Bourguignons. La maudite guerre, Paris, Libraire Académique Perrin, 1988.

Sheehan, M.M., The Formation and Stability of Marriage in Fourteenth-Century England: Evidence of an Ely Register, Mediaeval Studies, 1971, 33, p. 228-263.

Sheehan, M.M., Choice of Marriage Partner in the Middle Ages: Development and Mode of Application of a Theory of Marriage, Studies in Medieval and Renaissance History, 1978, 1, p. 1-33.

Turlan, J.M., Amis et amis chamels d'après les actes du Parlement au XIV siècle, Revue historique de droit français et étranger, 1969, 74, 4, p. 645-698.

Vantroys, A. Étude historique et juridique sur le consentement des parents au mariage, Paris, Arthur Rousseau, 1899. 